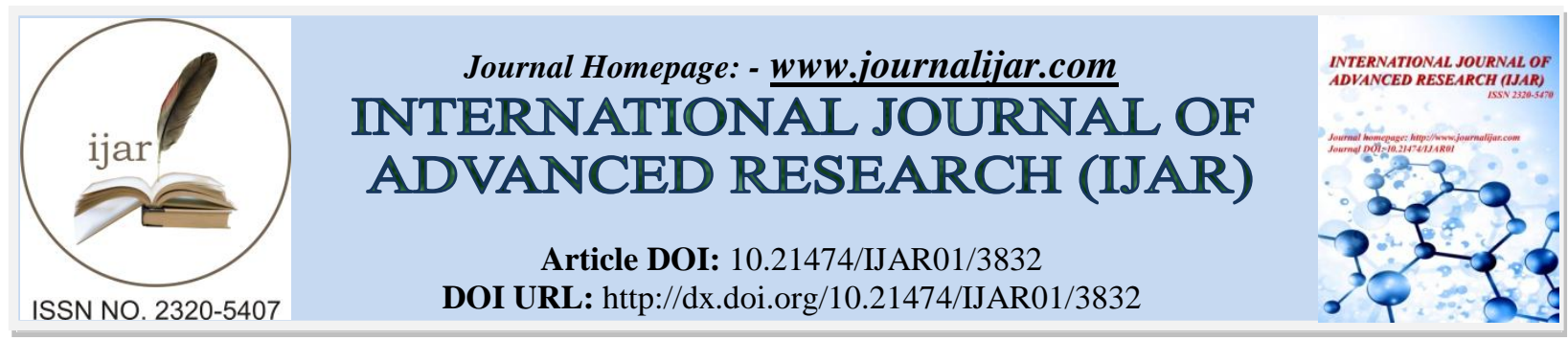

RESEARCH ARTICLE

\title{
TIME TO RECRUITMENT FOR A SINGLE GRADE MANPOWER SYSTEM WITH TWO TYPES OF LOSS OF MANPOWER AND THE BREAKDOWN THRESHOLD HAS TWO COMPONENTS.
}

\author{
P. Arokkia Saibe and B. Esther Clara.
}

PG \& Research Department of Mathematics, Bishop Heber College, Trichy- 620017, Tamil Nadu, India.

\section{Manuscript Info}

Manuscript History

Received: 07 February 2017

Final Accepted: 05 March 2017

Published: April 2017

Key words:-

Single grade manpower system, voluntary and involuntary exits, interexit times, two types of breaking decisions, univariate CUM policy of recruitment, variance of time to recruitment.

\begin{abstract}
In this paper, the problem of time to recruitment is studied for a single grade manpower system in which depletion of manpower takes place either by exit of personnel from the organization or due to breaks taken by the existing workers in the organization. The loss of manpower due to exits is classified as voluntary and involuntary. The breaking decisions are also classified into two types according to their frequency. It is also assumed that the breakdown threshold has two components, one for the cumulative loss of manpower due to exits and the other for the cumulative loss of manpower due to breaks. A Stochastic model is constructed for three different cases of inter-exit times and the variance of time to recruitment is obtained using a univariate CUM policy of recruitment. The results are numerically illustrated by assuming specific distributions. The influences of the nodal parameters on these system characteristics are studied and relevant conclusions are presented.
\end{abstract}

Copy Right, IJAR, 2017,. All rights reserved.

\section{Introduction:-}

Exit of personnel is a common phenomenon in any marketing organization. This leads to reduction in the total strength of marketing personnel and will adversely affect the sales turnover of the organization, if recruitment is not planned. Frequent recruitments may also be expensive due to the cost of recruitments and training. As the loss of manpower is unpredictable, suitable recruitment policy has to be designed to overcome this loss. An univariate recruitment policy, usually known as CUM policy of recruitment in the literature, is based on the replacement policy associated with the shock model approach in reliability theory is stated as follows: Recruitment is made whenever the cumulative loss of man hours exceeds a breakdown threshold.

Several stochastic models of manpower systems have been proposed and studied by several authors [1], [2], [3], [4] and [7] extensively in the past. More specifically [1] has studied many renewal theory models for several manpower systems in the context of filling up of vacancies in any grade of an organization either with promotions from next lower grade or by direct recruitment. [11] has initiated the study on finding the expected time to recruitment for a single grade manpower system using shock model approach in reliability theory [6]. Later, [8], [9], [10], [12] and [13] have studied the problem of time to recruitment under different conditions employing different recruitment policies such as univariate and bivariate policies of recruitment. Recently, [5] have studied this problem using a different probabilistic analysis by assuming that the policy decisions and exits occurs at different epochs. These authors have studied the problem of time to recruitment with the depletion of manpower which takes place only due to attrition, but there is another possible way that, depletion of manpower takes place due to breaks taken by the

Corresponding Author:- P. Arokkia Saibe.

Address:- PG \& Research Department of Mathematics, Bishop Heber College, Trichy- 620017, Tamil

Nadu, India. 
existing workers in the organization. In this context, the study in the problem of time to recruitment for a single grade manpower system is made in which (i) depletion of manpower takes place either by exit of personnel from the organization (voluntary or involuntary) or due to breaks taken by the existing workers in the organization, (ii) two types of decisions classified as policy decisions taken by the organization and breaking decisions taken by the personnel having low or high intensity of depletion and (iii) the breakdown threshold has two components, one component is for the exits of personnel from the organization and the another component is for depletion due to breaks are considered. In this paper, a stochastic model is constructed when the inter-exit times: (Case-I) forms a sequence of independent and identically distributed hyper exponential random variables, (Case-II) forms a sequence of exchangeable and constantly correlated exponential random variables and (Case-III) forms a geometric process and the variance of time to recruitment is obtained using a univariate CUM policy of recruitment by assuming specific distribution for the loss of manpower and thresholds. The influences of nodal parameters on these system characteristics are studied and relevant conclusions are presented.

\section{Model Description:-}

Consider an organization with single grade in which exit of personnel takes place either voluntarily (due to policy decisions, VRS etc.) or involuntarily (death, dismissal, retirement etc.). Let $X_{k}, k=1,2,3, \ldots$ be independent and identically distributed hyper-exponential random variables representing the amount of depletion of manpower in the organization due to $\mathrm{k}^{\text {th }}$ exit with probability distribution $F_{X}($.$) , density function f_{X}($.$) and Laplace transform \bar{f}_{X}($. with mean $\frac{P_{2}}{\alpha_{1}}+\frac{\left(1-P_{2}\right)}{\alpha_{2}}, \alpha_{1}, \alpha_{2}>0$ where $P_{2}$ is the proportion of involuntary loss of manpower with rate $\alpha_{1}$ and (1$P_{2}$ ) is the proportion of voluntary loss of manpower with rate $\alpha_{2}$. Let $\widetilde{X_{k}}$ be the cumulative loss of manpower in the first $\mathrm{k}$ exits of personnel from the organization with distribution function $F_{\widetilde{X_{k}}}($.$) , density function f_{\widetilde{X_{k}}}($.$) and$ Laplace transform $\bar{f}_{\widetilde{X}_{k}}($.$) . Let Y_{l}, l=1,2,3, \ldots$ be independent and identically distributed exponential random variables representing the amount of depletion of manpower in the organization due to $l^{\text {th }}$ break with probability distribution $G_{Y}($.$) , density function g_{Y}($.$) and Laplace transform \bar{g}_{Y}($.$) with mean \frac{1}{\gamma}, \gamma>0$. Let $\widetilde{G}_{l}$ be the cumulative loss of manpower in the first $l$ breaks with distribution function $G_{\widetilde{Y_{l}}}($.$) , density function g_{\widetilde{Y_{l}}}($.$) and Laplace transform$ $\bar{g}_{\widetilde{Y}_{l}}($.$) . Let \mathrm{U}_{\mathrm{k}}, \mathrm{k}=1,2,3, \ldots$ be independent and identically distributed hyper-exponential random variables representing the time between $\mathrm{k}-1^{\text {th }}$ and $\mathrm{k}^{\text {th }}$ exit of personnel from the organization with probability distribution $F_{U}($.$) , density function f_{U}($.$) and Laplace transform \bar{f}_{U}($.$) with mean \frac{P_{1}}{\lambda_{1}}+\frac{\left(1-P_{1}\right)}{\lambda_{2}}, \lambda_{1}, \lambda_{2}>0$ where $P_{1}$ is the proportion of exits having high attrition rate $\lambda_{1}$ and $\left(1-P_{1}\right)$ is the proportion of exits having low attrition rate $\lambda_{2}$. Let $V_{l}, l=1,2,3, \ldots$ be independent and identically distributed hyper-exponential random variables representing the time between $l-1^{\text {th }}$ and $l^{\text {th }}$ break with probability distribution $G_{V}($.$) and density function g_{V}($.$) with mean \frac{P_{3}}{\beta_{1}}+\frac{\left(1-P_{3}\right)}{\beta_{2}}$, $\beta_{1}, \beta_{2}>0$ where $P_{3}$ is the proportion of frequent breaks having high rate of depletion $\beta_{1}$ and $\left(1-P_{3}\right)$ is the proportion of non-frequent breaks having low rate of depletion $\beta_{2}$. Let $N_{1}(t)$ be the number of exit decisions in $(0, \mathrm{t}]$ and $N_{2}(t)$ be the number of breaking decisions in $(0, t]$. Let $Z_{1}$ be the threshold for the cumulative loss of manpower due to exits of personnel from the organization with parameter $\theta_{E}>0$ and $Z_{2}$ be the threshold for the cumulative loss of manpower due to breaks with parameter $\theta_{B}>0$. Let $Z_{1}+Z_{2}$ be the breakdown threshold for the organization. Let $\mathrm{W}$ be the time to recruitment for the organization with the distribution function $\mathrm{L}($.), density function $1($.$) and the Laplace transform \bar{l}($.$) .$

\section{Main Results:-}

The event of time to recruitment is defined as follows: recruitment occurs beyond $t$ if and only if the total loss of manpower due to $N_{1}(t)$ exit decisions and $N_{2}(t)$ breaking decisions does not crossed the breakdown threshold of the organization and it is written as follows

$(\mathrm{W}>\mathrm{t})=\left(\widetilde{X_{N_{1}(t)}}+\widetilde{Y_{N_{2}}(t)}<Z_{1}+Z_{2}\right)$

Hence the probabilities of the occurrence of these two events are equal. Now, conditioning upon $N_{1}(t)$ and $N_{2}(t)$ the distribution of time to recruitment is determined.The $\mathrm{r}^{\text {th }}$ moment for the time to recruitment is determined by taking the $\mathrm{r}^{\text {th }}$ derivative of the Laplace transform of density function for the random variable with respect to $s$, and at $s=0$. Using this result the mean and variance of time to recruitment is determined for the three different cases of inter-exit times. 
Case-I: Inter-exit times are assumed to form a sequence of independent and identically distributed hyperexponential random variables. Using the tool of finding the moments of time to recruitment, the mean time to recruitment is given as

$\mathrm{E}(\mathrm{W})=\frac{\theta_{B}}{\theta_{B}-\theta_{E}} D_{1}-\frac{\theta_{E}}{\theta_{B}-\theta_{E}} D_{2}$

where

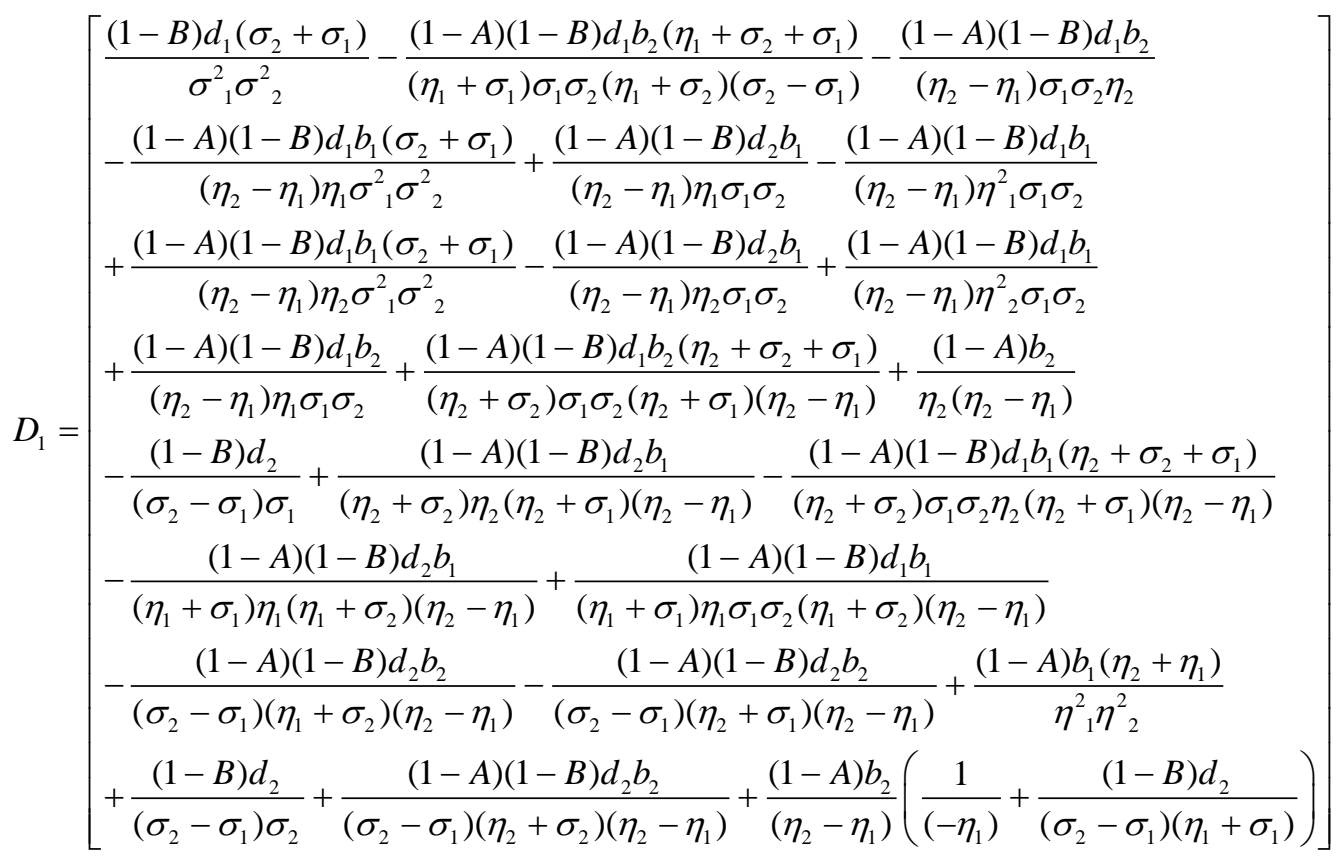

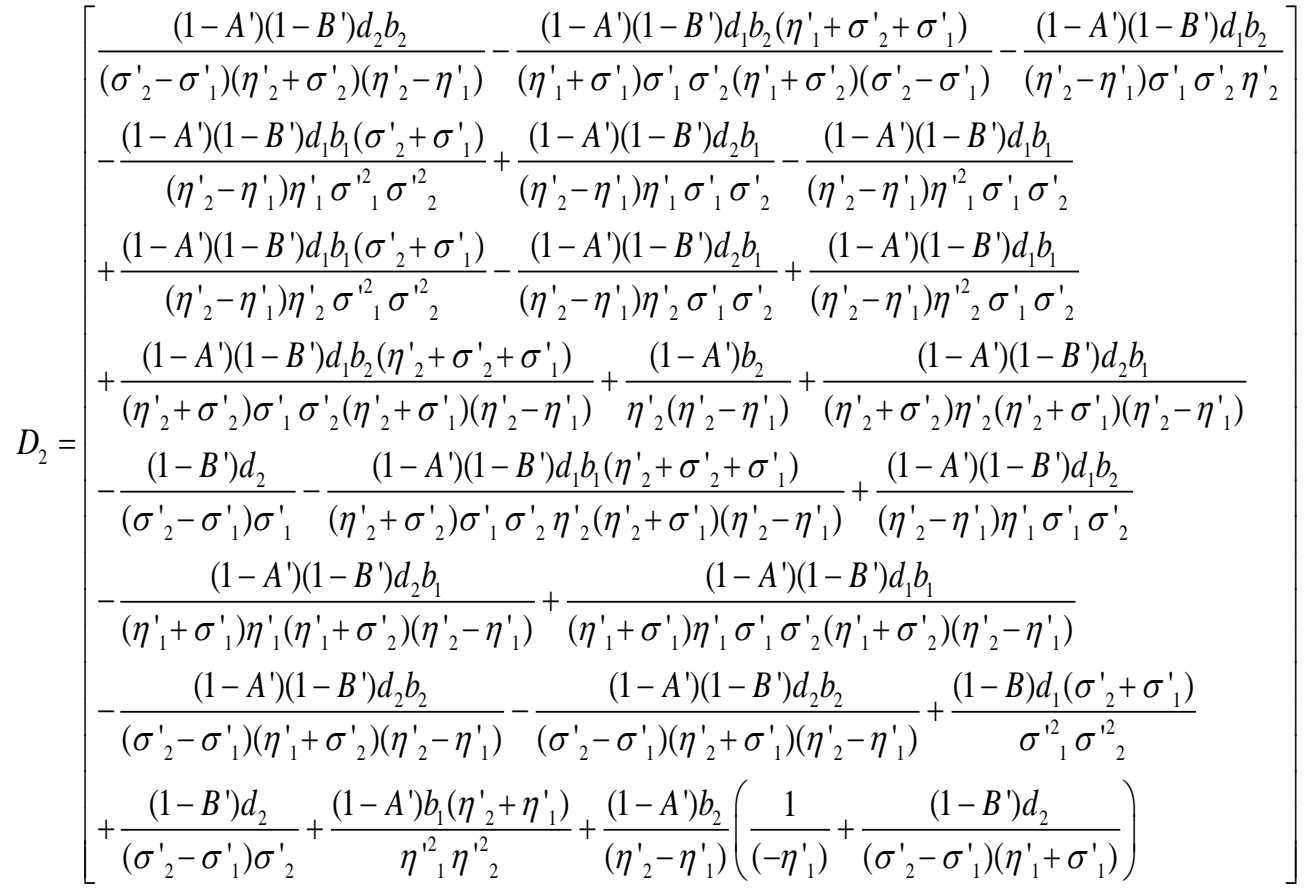

Again differentiating twice with respect to $\mathrm{s}$, the Laplace transform of the density function of time to recruitment at $\mathrm{s}=0$, the second moment for $\mathrm{W}$ is given by 
$\mathrm{E}\left(\mathrm{W}^{2}\right)=\frac{\theta_{B}}{\theta_{B}-\theta_{E}} D_{1}^{2}-\frac{\theta_{E}}{\theta_{B}-\theta_{E}} D_{2}^{2}$

where

$$
\begin{aligned}
& {\left[\frac{(1-A) b_{1}\left(\eta_{2}^{2}+\eta_{2} \eta_{1}+\eta_{1}^{2}\right)}{\eta_{2}^{3} \eta_{1}^{3}}-\frac{(1-A)(1-B) d_{1} b_{2}\left(\eta_{1}^{2}+\left(2 \eta_{1}+\sigma_{2}\right)\left(\sigma_{2}+\sigma_{1}\right)+\sigma_{1}^{2}\right)}{\left(\eta_{1}+\sigma_{1}\right)^{2} \sigma_{1} \sigma_{2}\left(\eta_{1}+\sigma_{2}\right)^{2}\left(\eta_{2}-\eta_{1}\right)}-\frac{(1-A)(1-B) d_{1} b_{2}}{\left(\eta_{2}-\eta_{1}\right) \sigma_{1} \sigma_{2} \eta_{2}^{2}}\right.} \\
& -\frac{(1-B) d_{2}}{\sigma_{1}^{2}\left(\sigma_{2}-\sigma_{1}\right)}+\frac{(1-B) d_{1}\left(\sigma_{2}^{2}+\sigma_{2} \sigma_{1}+\sigma_{1}^{2}\right)}{\sigma_{2}^{3} \sigma_{1}^{3}}-\frac{(1-B)(1-A) b_{1} d_{1}\left(\sigma_{2}^{2}+\sigma_{2} \sigma_{1}+\sigma_{1}^{2}\right)}{\sigma_{1}^{3}\left(\eta_{2}-\eta_{1}\right) \eta_{1}}+\frac{(1-B)(1-A) b_{1} d_{2}\left(\sigma_{2}+\sigma_{1}\right)}{\sigma_{1}^{2} \sigma_{2}^{2}\left(\eta_{2}-\eta_{1}\right) \eta_{1}} \\
& -\frac{(1-B)(1-A) b_{1} d_{1}}{\sigma_{1} \sigma_{2}\left(\eta_{2}-\eta_{1}\right) \eta_{1}{ }^{3}}+\frac{(1-B)(1-A) b_{1} d_{1}\left(\sigma_{2}^{2}+\sigma_{2} \sigma_{1}+\sigma_{1}^{2}\right)}{\sigma_{1}^{2} \sigma_{2}^{2}\left(\eta_{2}-\eta_{1}\right) \eta_{2}}-\frac{(1-B)(1-A) b_{1} d_{2}\left(\sigma_{2}+\sigma_{1}\right)}{\sigma_{1}^{2} \sigma_{2}^{2}\left(\eta_{2}-\eta_{1}\right) \eta_{2}}+\frac{(1-B)(1-A) b_{1} d_{1}}{\sigma_{1} \sigma_{2}\left(\eta_{2}-\eta_{1}\right) \eta_{2}{ }^{3}} \\
& D_{1}^{2}=+\frac{(1-B)(1-A) b_{2} d_{1}}{\sigma_{1} \sigma_{2}\left(\eta_{2}-\eta_{1}\right) \eta_{1}^{2}}+\frac{(1-A)(1-B) d_{1} b_{2}\left(\eta_{2}^{2}+\left(2 \eta_{2}+\sigma_{2}\right)\left(\sigma_{2}+\sigma_{1}\right)+\sigma_{1}^{2}\right)}{\left(\eta_{2}+\sigma_{1}\right)^{2} \sigma_{1} \sigma_{2}\left(\eta_{2}+\sigma_{2}\right)^{2}\left(\eta_{2}-\eta_{1}\right)}+\frac{(1-A)(1-B) d_{2} b_{1}\left(2 \eta_{2}+\sigma_{2}+\sigma_{1}\right)}{\left(\eta_{2}+\sigma_{2}\right)^{2} \eta_{2}\left(\eta_{2}+\sigma_{1}\right)^{2}\left(\eta_{2}-\eta_{1}\right)} \\
& +\frac{(1-A) b_{2}}{\eta_{2}^{2}\left(\eta_{2}-\eta_{1}\right)}-\frac{(1-A)(1-B) d_{1} b_{1}\left(\eta_{2}^{2}+\left(2 \eta_{2}+\sigma_{2}\right)\left(\sigma_{2}+\sigma_{1}\right)+\sigma_{1}^{2}\right)}{\eta_{2}\left(\eta_{2}+\sigma_{1}\right)^{2} \sigma_{1} \sigma_{2}\left(\eta_{2}+\sigma_{2}\right)^{2}\left(\eta_{2}-\eta_{1}\right)}-\frac{(1-A)(1-B) d_{2} b_{1}\left(2 \eta_{1}+\sigma_{2}+\sigma_{1}\right)}{\left(\eta_{1}+\sigma_{2}\right)^{2} \eta_{1}\left(\eta_{1}+\sigma_{1}\right)^{2}\left(\eta_{2}-\eta_{1}\right)} \\
& +\frac{(1-B) d_{2}}{\sigma_{2}^{2}\left(\sigma_{2}-\sigma_{1}\right)}-\frac{(1-A)(1-B) d_{2} b_{2}}{\left(\eta_{2}+\sigma_{1}\right)^{2}\left(\eta_{2}-\eta_{1}\right)\left(\sigma_{2}-\sigma_{1}\right)}+\frac{(1-A)(1-B) d_{2} b_{2}}{\left(\eta_{2}+\sigma_{2}\right)^{2}\left(\eta_{2}-\eta_{1}\right)\left(\sigma_{2}-\sigma_{1}\right)}+\frac{(1-A) b_{2}}{\left(\eta_{2}-\eta_{1}\right)} \\
& {\left[\left(\frac{1}{\left(-\eta_{1}^{2}\right)}+\frac{(1-B) d_{2}}{\left(\sigma_{2}-\sigma_{1}\right)\left(\eta_{1}+\sigma_{1}\right)^{2}}\right)-\frac{(1-A)(1-B) d_{2} b_{2}}{\left(\eta_{1}+\sigma_{2}\right)^{2}\left(\eta_{2}-\eta_{1}\right)\left(\sigma_{2}-\sigma_{1}\right)}+\frac{(1-A)(1-B) d_{1} b_{1}\left(\eta_{1}^{2}+\left(2 \eta_{1}+\sigma_{2}\right)\left(\sigma_{2}+\sigma_{1}\right)+\eta_{1}^{2}\right)}{\left(\eta_{1}+\sigma_{1}\right)^{2} \sigma_{1} \eta_{1} \sigma_{2}\left(\eta_{1}+\sigma_{2}\right)^{2}\left(\eta_{2}-\eta_{1}\right)}\right]} \\
& {\left[\begin{array}{l}
\left(1-A^{\prime}\right. \\
1
\end{array}\right.} \\
& \frac{\left(1-A^{\prime}\right) b_{1}\left(\eta_{2}^{\prime}{ }_{2}^{2}+\eta_{2}^{\prime} \eta_{1}^{\prime}+\eta_{1}^{\prime 2}\right)}{\eta_{2}^{\prime 3} \eta_{1}^{\prime 3}}-\frac{\left(1-A^{\prime}\right)\left(1-B^{\prime}\right) d_{1} b_{2}\left(\eta_{1}^{\prime 2}+\left(2 \eta_{1}^{\prime}+\sigma_{2}^{\prime}\right)\left(\sigma_{2}^{\prime}+\sigma_{1}^{\prime}\right)+\sigma_{1}^{\prime 2}\right)}{\left(\eta_{1}^{\prime}+\sigma_{1}^{\prime}\right)^{2} \sigma_{1}^{\prime} \sigma_{2}^{\prime}\left(\eta_{1}^{\prime}+\sigma_{2}^{\prime}\right)^{2}\left(\eta_{2}^{\prime}-\eta_{1}^{\prime}\right)}-\frac{\left(1-A^{\prime}\right)\left(1-B^{\prime}\right) d_{1} b_{2}}{\left(\eta_{2}^{\prime}-\eta_{1}^{\prime}\right) \sigma_{1}^{\prime} \sigma_{2}^{\prime} \eta_{2}^{\prime 2}} \\
& +\frac{\left(1-B^{\prime}\right) d_{1}\left(\sigma_{2}^{\prime 2}+\sigma_{2}^{\prime} \sigma_{1}^{\prime}+\sigma_{1}^{\prime 2}\right)}{\sigma_{2}^{\prime 3} \sigma_{1}^{\prime 3}}-\frac{\left(1-B^{\prime}\right)\left(1-A^{\prime}\right) b_{1} d_{1}\left(\sigma_{2}^{\prime 2}+\sigma_{2}^{\prime} \sigma_{1}^{\prime}+\sigma_{1}^{\prime 2}\right)}{\sigma_{1}^{\prime 3}\left(\eta_{2}^{\prime}-\eta_{1}^{\prime}\right) \eta_{1}^{\prime}}+\frac{\left(1-B^{\prime}\right)\left(1-A^{\prime}\right) b_{1} d_{2}\left(\sigma_{2}^{\prime}+\sigma_{1}^{\prime}\right)}{\sigma_{1}^{\prime 2} \sigma_{2}^{\prime 2}\left(\eta_{2}^{\prime}-\eta_{1}^{\prime}\right) \eta_{1}^{\prime}} \\
& +\frac{\left(1-B^{\prime}\right) d_{2}}{\sigma_{2}^{\prime 2}\left(\sigma_{2}^{\prime}-\sigma_{1}^{\prime}\right)}+\frac{\left(1-B^{\prime}\right)\left(1-A^{\prime}\right) b_{1} d_{1}\left(\sigma_{2}^{\prime 2}+\sigma_{2}^{\prime} \sigma_{1}^{\prime}+\sigma_{1}^{\prime 2}\right)}{\sigma_{1}^{\prime 2} \sigma_{2}^{\prime 2}\left(\eta_{2}^{\prime}-\eta_{1}^{\prime}\right) \eta_{2}^{\prime}}-\frac{\left(1-B^{\prime}\right)\left(1-A^{\prime}\right) b_{1} d_{2}\left(\sigma_{2}^{\prime}+\sigma_{1}^{\prime}\right)}{\sigma_{1}^{\prime 2} \sigma_{2}^{\prime 2}\left(\eta_{2}^{\prime}-\eta_{1}^{\prime}\right) \eta_{2}^{\prime}}+\frac{\left(1-B^{\prime}\right)\left(1-A^{\prime}\right) b_{1} d_{1}}{\sigma_{1}^{\prime} \sigma_{2}^{\prime}\left(\eta_{2}^{\prime}-\eta_{1}^{\prime}\right) \eta_{2}^{\prime 3}} \\
& D_{2}^{2}=+\frac{\left(1-B^{\prime}\right)\left(1-A^{\prime}\right) b_{2} d_{1}}{\sigma_{1}^{\prime} \sigma_{2}^{\prime}\left(\eta_{2}^{\prime}-\eta_{1}^{\prime}\right) \eta_{1}^{\prime 2}}+\frac{\left(1-A^{\prime}\right)\left(1-B^{\prime}\right) d_{1} b_{2}\left(\eta_{2}^{\prime}{ }_{2}+\left(2 \eta_{2}^{\prime}+\sigma_{2}^{\prime}\right)\left(\sigma_{2}^{\prime}+\sigma_{1}^{\prime}\right)+\sigma_{1}^{\prime 2}\right)}{\left(\eta_{2}^{\prime}+\sigma_{1}^{\prime}\right)^{2} \sigma_{1}^{\prime} \sigma_{2}^{\prime}\left(\eta_{2}^{\prime}+\sigma_{2}^{\prime}\right)^{2}\left(\eta_{2}^{\prime}-\eta_{1}^{\prime}\right)}+\frac{\left(1-A^{\prime}\right)\left(1-B^{\prime}\right) d_{2} b_{1}\left(2 \eta_{2}^{\prime}+\sigma_{2}^{\prime}+\sigma_{1}^{\prime}\right)}{\left(\eta_{2}^{\prime}+\sigma_{2}^{\prime}\right)^{2} \eta_{2}^{\prime}{ }_{2}\left(\eta_{2}^{\prime}+\sigma_{1}^{\prime}\right)^{2}\left(\eta_{2}^{\prime}-\eta_{1}^{\prime}\right)} \\
& +\frac{\left(1-A^{\prime}\right) b_{2}}{\eta_{2}^{\prime}{ }_{2}^{2}\left(\eta_{2}^{\prime}-\eta_{1}^{\prime}\right)}-\frac{\left(1-A^{\prime}\right)\left(1-B^{\prime}\right) d_{1} b_{1}\left(\eta_{2}^{\prime}{ }_{2}+\left(2 \eta_{2}^{\prime}+\sigma_{2}^{\prime}\right)\left(\sigma_{2}^{\prime}+\sigma_{1}^{\prime}\right)+\sigma_{1}^{\prime 2}\right)}{\eta_{2}^{\prime}\left(\eta_{2}^{\prime}+\sigma_{1}^{\prime}\right)^{2} \sigma_{1}^{\prime} \sigma_{2}^{\prime}\left(\eta_{2}^{\prime}{ }_{2}+\sigma_{2}^{\prime}\right)^{2}\left(\eta_{2}^{\prime}-\eta_{1}^{\prime}\right)}-\frac{\left(1-A^{\prime}\right)\left(1-B^{\prime}\right) d_{2} b_{1}\left(2 \eta_{1}^{\prime}+\sigma_{2}^{\prime}+\sigma_{1}^{\prime}\right)}{\left(\eta_{1}^{\prime}+\sigma_{2}^{\prime}\right)^{2} \eta_{1}^{\prime}\left(\eta_{1}^{\prime}+\sigma_{1}^{\prime}\right)^{2}\left(\eta_{2}^{\prime}-\eta_{1}^{\prime}\right)} \\
& {\left[\begin{array}{l}
-\frac{\left(1-B^{\prime}\right)\left(1-A^{\prime}\right) b_{1} d_{1}}{\sigma_{1}^{\prime} \sigma_{2}^{\prime}\left(\eta_{2}^{\prime}-\eta_{1}^{\prime}\right) \eta_{1}^{\prime 3}}-\frac{\left(1-A^{\prime}\right)\left(1-B^{\prime}\right) d_{2} b_{2}}{\left(\eta_{2}^{\prime}+\sigma_{1}^{\prime}\right)^{2}\left(\eta_{2}^{\prime}-\eta_{1}^{\prime}\right)\left(\sigma_{2}^{\prime}-\sigma_{1}^{\prime}\right)}+\frac{\left(1-A^{\prime}\right)\left(1-B^{\prime}\right) d_{2} b_{2}}{\left(\eta_{2}^{\prime}+\sigma_{2}^{\prime}\right)^{2}\left(\eta_{2}^{\prime}-\eta_{1}^{\prime}\right)\left(\sigma_{2}^{\prime}-\sigma_{1}^{\prime}\right)}+\frac{\left(1-A^{\prime}\right) b_{2}}{\left(\eta_{2}^{\prime}-\eta_{1}^{\prime}\right)}\left(\begin{array}{l}
\frac{1}{\left(-\eta_{1}^{\prime 2}\right)}+ \\
\frac{\left(1-B^{\prime}\right) d_{2}}{\left(\sigma_{2}^{\prime}-\sigma_{1}^{\prime}\right)\left(\eta_{1}^{\prime}+\sigma_{1}^{\prime}\right)^{2}}
\end{array}\right. \\
-\frac{\left(1-B^{\prime}\right) d_{2}}{\sigma_{1}^{\prime 2}\left(\sigma_{2}^{\prime}-\sigma_{1}^{\prime}\right)}-\frac{\left(1-A^{\prime}\right)\left(1-B^{\prime}\right) d_{2} b_{2}}{\left(\eta_{1}^{\prime}+\sigma_{2}^{\prime}\right)^{2}\left(\eta_{2}^{\prime}-\eta_{1}^{\prime}\right)\left(\sigma_{2}^{\prime}-\sigma_{1}^{\prime}\right)}+\frac{\left(1-A^{\prime}\right)\left(1-B^{\prime}\right) d_{1} b_{1}\left(\eta_{1}^{\prime}{ }_{1}^{2}+\left(2 \eta_{1}^{\prime}+\sigma_{2}^{\prime}\right)\left(\sigma_{2}^{\prime}+\sigma_{1}^{\prime}\right)+\eta_{1}^{\prime 2}\right)}{\left(\eta_{1}^{\prime}+\sigma_{1}^{\prime}\right)^{2} \sigma_{1}^{\prime} \eta_{1}^{\prime} \sigma_{2}^{\prime}\left(\eta_{1}^{\prime}+\sigma_{2}^{\prime}\right)^{2}\left(\eta_{2}^{\prime}-\eta_{1}^{\prime}\right)}
\end{array}\right]}
\end{aligned}
$$

Using equations (2) and (3), the variance of time to recruitment for the organization is obtained.

\section{Remark:}

When $\alpha_{2}=\lambda_{2}=\beta_{2}=0$ and $P_{1}=P_{2}=P_{3}=1$ the distribution of the depletion of manpower, inter-exit times and inter-breaking decision times reduces to exponential distribution. The mean and variance of the time to recruitment can be deduced for the above case from the results of (2) and (3).

Case-II: In this case inter-exit times are assumed to form a sequence of exchangeable and constantly correlated exponential random variables with mean $\lambda$, correlation $\rho \in(-1,1)$ with relation $\mathrm{v}=\lambda(1-\rho)$.

Now, differentiating with respect to $s$, the Laplace transform of the density function $\mathrm{W}$ and at $\mathrm{s}=0$ the mean time to recruitment is given by

$\mathrm{E}(\mathrm{W})=\frac{\theta_{B}}{\theta_{B}-\theta_{E}} D_{3}-\frac{\theta_{E}}{\theta_{B}-\theta_{E}} D_{4}$ 
where

$$
D_{3}=\left(\begin{array}{l}
\frac{(1-B) d_{1}\left(\sigma_{2}+\sigma_{1}\right)}{\sigma_{1}^{2} \sigma_{2}^{2}}-\frac{(1-B) d_{2}}{\sigma_{1} \sigma_{2}}-\frac{(1-B) d_{1} \lambda}{\left(\sigma_{2}-\sigma_{1}\right) \sigma_{1}(1-A)}+\frac{\lambda}{(1-A)}+\frac{(1-B) d_{1} \lambda}{\left(\sigma_{2}-\sigma_{1}\right) \sigma_{2}(1-A)} \\
-\frac{(1-B) d_{1}(1-A)}{\left(\sigma_{2}-\sigma_{1}\right) \sigma_{1}^{2}} \sum_{k=1}^{\infty} \frac{(1-\rho)\left(1+v \sigma_{1}\right)^{1-k} A^{k-1}}{(1-\rho)\left(1+v \sigma_{1}\right)+k \rho v \sigma_{1}}+\frac{(1-B) d_{1}(1-A)}{\left(\sigma_{2}-\sigma_{1}\right) \sigma_{2}^{2}} \sum_{k=1}^{\infty} \frac{(1-\rho)\left(1+v \sigma_{2}\right)^{1-k} A^{k-1}}{(1-\rho)\left(1+v \sigma_{2}\right)+k \rho v \sigma_{2}}
\end{array}\right)
$$

and

$$
D_{4}=\left(\begin{array}{l}
\frac{\left(1-B^{\prime}\right) d_{1}\left(\sigma_{2}^{\prime}+\sigma_{1}^{\prime}\right)}{\sigma_{1}^{\prime 2} \sigma_{2}^{\prime 2}}-\frac{\left(1-B^{\prime}\right) d_{1} \lambda}{\left(\sigma_{2}^{\prime}-\sigma_{1}^{\prime}\right) \sigma_{1}^{\prime}\left(1-A^{\prime}\right)}-\frac{\left(1-B^{\prime}\right) d_{1}\left(1-A^{\prime}\right)}{\left(\sigma_{2}^{\prime}-\sigma_{1}^{\prime}\right) \sigma_{1}^{\prime 2}} \sum_{k=1}^{\infty} \frac{(1-\rho)\left(1+v \sigma_{1}^{\prime}\right)^{1-k} A^{\prime k-1}}{(1-\rho)\left(1+v \sigma_{1}^{\prime}\right)+k \rho v \sigma_{1}^{\prime}} \\
+\frac{\left(1-B^{\prime}\right) d_{1}\left(1-A^{\prime}\right)}{\left(\sigma_{2}^{\prime}{ }_{2}-\sigma_{1}^{\prime}\right) \sigma_{2}^{\prime}{ }_{2}^{2}} \sum_{k=1}^{\infty} \frac{(1-\rho)\left(1+v \sigma_{2}^{\prime}\right)^{1-k} A^{\prime k-1}}{(1-\rho)\left(1+v \sigma_{2}^{\prime}\right)+k \rho v \sigma_{2}^{\prime}}+\frac{\lambda}{\left(1-A^{\prime}\right)}+\frac{\left(1-B^{\prime}\right) d_{1} \lambda}{\left(\sigma_{2}^{\prime}{ }_{2}-\sigma_{1_{1}}\right) \sigma_{2}^{\prime}\left(1-A^{\prime}\right)}-\frac{\left(1-B^{\prime}\right) d_{2}}{\sigma_{1}^{\prime} \sigma_{1}^{\prime}{ }_{2}}
\end{array}\right)
$$

Now, differentiating twice with respect to $\mathrm{s}$, the Laplace transform of the density function $\mathrm{W}$ at $\mathrm{s}=0$ gives the second moment of $\mathrm{W}$.

$$
\mathrm{E}\left(\mathrm{W}^{2}\right)=\frac{\theta_{B}}{\theta_{B}-\theta_{E}} D_{3}{ }^{2}-\frac{\theta_{E}}{\theta_{B}-\theta_{E}} D_{4}{ }^{2}
$$

where

$$
D_{3}^{2}=\left(\begin{array}{l}
\frac{2(1-B) d_{1}}{\left(\sigma_{2}-\sigma_{1}\right) \sigma_{1}^{3}}-\frac{2(1-B) d_{1}}{\left(\sigma_{2}-\sigma_{1}\right) \sigma_{2}^{3}}-\frac{2(1-B) d_{2}}{\left(\sigma_{2}-\sigma_{1}\right) \sigma_{1}^{2}}+\frac{2(1-B) d_{2}}{\left(\sigma_{2}-\sigma_{1}\right) \sigma_{2}^{2}}-\frac{2(1-B) d_{1} \lambda^{2}}{\left(\sigma_{2}-\sigma_{1}\right)}\left[\frac{1+\rho^{2} A}{\sigma_{1}(1-A)^{2}}-\frac{1+\rho^{2} A}{\sigma_{2}(1-A)^{2}}\right] \\
+\frac{2\left(1+\rho^{2} A\right) \lambda^{2}}{(1-A)^{2}}-\frac{2(1-A)(1-B) d_{1}}{\left(\sigma_{2}-\sigma_{1}\right)}\left[\begin{array}{l}
\sum_{k=1}^{\infty}-\frac{v(1-\rho)\left(1+v \sigma_{2}\right)^{-k}\left(v \sigma_{2}[1-k(1-\rho+k \rho)]-k\right) A^{k-1}}{\sigma_{2}^{2}\left(1-\rho+v \sigma_{2}[1-\rho+k \rho]\right)^{2}} \\
\sigma_{1}^{2}\left(1-\rho+v \sigma_{1}[1-\rho+k \rho]\right)^{2}
\end{array}\right] \\
-\frac{2(1-A)(1-B) d_{1}}{\left(\sigma_{2}-\sigma_{1}\right)}\left[\sum_{k=1}^{\infty} \frac{(1-\rho)\left(1+v \sigma_{1}\right)^{1-k} A^{k-1}}{\left((1-\rho)\left(1+v \sigma_{1}\right)+k \rho v \sigma_{1}\right) \sigma_{1}^{3}}-\sum_{k=1}^{\infty} \frac{(1-\rho)\left(1+v \sigma_{2}\right)^{1-k} A^{k-1}}{\left((1-\rho)\left(1+v \sigma_{2}\right)+k \rho v \sigma_{2}\right) \sigma_{2}^{3}}\right]
\end{array}\right)
$$

and

$$
\begin{aligned}
& \left(\frac{2\left(1-B^{\prime}\right) d_{1}}{\left(\sigma_{2}^{\prime}-\sigma_{1}^{\prime}\right) \sigma_{1}^{\prime 3}}-\frac{2\left(1-B^{\prime}\right) d_{1}}{\left(\sigma_{2}^{\prime}-\sigma_{1}^{\prime}\right) \sigma_{2}^{\prime 3}}-\frac{2\left(1-B^{\prime}\right) d_{2}}{\left(\sigma_{2}^{\prime}-\sigma_{1}^{\prime}\right) \sigma_{1}^{\prime 2}}+\frac{2\left(1-B^{\prime}\right) d_{2}}{\left(\sigma_{2}^{\prime}-\sigma_{1}^{\prime}\right) \sigma_{2}^{\prime 2}}-\frac{2\left(1-B^{\prime}\right) d_{1} \lambda^{2}}{\left(\sigma_{2}^{\prime}-\sigma_{1}^{\prime}\right)}\left[\frac{1+\rho^{2} A^{\prime}}{\sigma_{1}^{\prime}\left(1-A^{\prime}\right)^{2}}-\frac{1+\rho^{2} A^{\prime}}{\sigma_{2}^{\prime}\left(1-A^{\prime}\right)^{2}}\right]\right)
\end{aligned}
$$

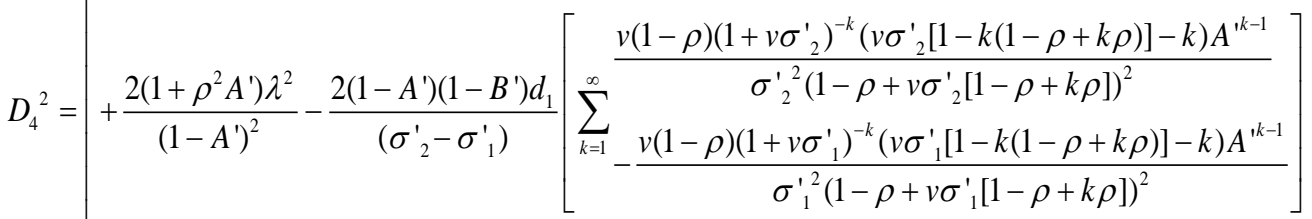

$$
\begin{aligned}
& -\frac{2\left(1-A^{\prime}\right)\left(1-B^{\prime}\right) d_{1}}{\left(\sigma_{2}^{\prime}-\sigma_{1}^{\prime}\right)}\left[\sum_{k=1}^{\infty} \frac{(1-\rho)\left(1+v \sigma_{1}^{\prime}\right)^{1-k} A^{\prime k-1}}{\left((1-\rho)\left(1+v \sigma_{1}^{\prime}\right)+k \rho v \sigma_{1}^{\prime}\right) \sigma_{1}^{\prime 3}}-\sum_{k=1}^{\infty} \frac{(1-\rho)\left(1+v \sigma_{2}^{\prime}\right)^{1-k} A^{\prime k-1}}{\left((1-\rho)\left(1+v \sigma_{2}^{\prime}\right)+k \rho v \sigma_{2}^{\prime}\right) \sigma_{2}^{\prime 3}}\right]
\end{aligned}
$$

For the present Case, using equations (4) and (5), the variance of time to recruitment for the organization is determined.

Case-III: In this case inter-exit times are assumed to form a geometric process with a parameter a>0.

Using the tool of deriving the moments of time to recruitment, the mean time to recruitment for Case-III is determined, by differentiating with respect to $s$, the Laplace transform of the density function of the random variable $\mathrm{W}$ at $\mathrm{s}=0$.

$\mathrm{E}(\mathrm{W})=\frac{\theta_{B}}{\theta_{B}-\theta_{E}} D_{5}-\frac{\theta_{E}}{\theta_{B}-\theta_{E}} D_{6}$

where 


$$
\begin{aligned}
& \left(\frac{(1-B) d_{1}\left(\sigma_{2}+\sigma_{1}\right)}{\sigma_{1}^{2} \sigma_{2}^{2}}-\frac{(1-B) d_{2}}{\sigma_{1} \sigma_{2}}-\frac{\bar{f}^{\prime}(0)}{(a-A)}+\frac{\left(1-B^{\prime} d_{1} \bar{f}^{\prime}(0)\right.}{\left(\sigma_{2}-\sigma_{1}\right) \sigma_{1}(a-A)}-\frac{(1-B) d_{1} \bar{f}^{\prime}(0)}{\left(\sigma_{2}-\sigma_{1}\right) \sigma_{2}(a-A)}-\right) \\
& D_{5}=\left[\frac{(1-B) d_{1}(1-A)}{\left(\sigma_{2}-\sigma_{1}\right) \sigma_{1}^{2}}\left[\begin{array}{l}
\sum_{k=1}^{\infty} \bar{f}_{U_{1}}^{\prime}\left(\sigma_{1}\right) \prod_{j=2}^{k} \bar{f}_{U_{j}}\left(\frac{\sigma_{1}}{a^{j-1}}\right)+\bar{f}_{U_{2}}^{\prime}\left(\frac{\sigma_{1}}{a}\right)\left(\frac{1}{a}\right) \prod_{j=1, j \neq 2}^{k} \bar{f}_{U_{j}}\left(\frac{\sigma_{1}}{a^{j-1}}\right)+\ldots \\
+\bar{f}_{U_{k}}^{\prime}\left(\frac{\sigma_{1}}{a^{k-1}}\right)\left(\frac{1}{a^{k-1}}\right) \prod_{j=1}^{k-1} \bar{f}_{U_{j}}\left(\frac{\sigma_{1}}{a^{j-1}}\right)
\end{array}\right] A^{k-1}\right. \\
& \left.+\frac{(1-B) d_{1}(1-A)}{\left(\sigma_{2}-\sigma_{1}\right) \sigma_{2}^{2}}\left[\begin{array}{l}
\sum_{k=1}^{\infty} \bar{f}_{U_{1}}{ }^{\prime}\left(\sigma_{2}\right) \prod_{j=2}^{k} \bar{f}_{U_{j}}\left(\frac{\sigma_{2}}{a^{j-1}}\right)+\bar{f}_{U 2}\left(\frac{\sigma_{2}}{a}\right)\left(\frac{1}{a}\right) \prod_{j=1, j \neq 2}^{k} \bar{f}_{U_{j}}\left(\frac{\sigma_{2}}{a^{j-1}}\right)+. . \\
++\bar{f}_{U_{k}}^{\prime}\left(\frac{\sigma_{2}}{a^{k-1}}\right)\left(\frac{1}{a^{k-1}}\right) \prod_{j=1}^{k-1} \bar{f}_{U_{j}}\left(\frac{\sigma_{2}}{a^{j-1}}\right)
\end{array}\right] A^{k-1}\right] \\
& \left(\frac{\left(1-B^{\prime}\right) d_{1}\left(\sigma_{2}^{\prime}+\sigma_{1}^{\prime}\right)}{\sigma_{1}^{\prime 2} \sigma_{2}^{\prime 2}}-\frac{\left(1-B^{\prime}\right) d_{2}}{\sigma_{1}^{\prime} \sigma_{2}^{\prime}}-\frac{\bar{f}^{\prime}(0)}{\left(a-A^{\prime}\right)}+\frac{\left(1-B^{\prime}\right) d_{1} \bar{f}^{\prime}(0)}{\left(\sigma_{2}^{\prime}-\sigma_{1}^{\prime}\right) \sigma_{1}^{\prime}\left(a-A^{\prime}\right)}-\frac{\left(1-B^{\prime}\right) d_{1} \bar{f}^{\prime}(0)}{\left(\sigma_{2}^{\prime}-\sigma_{1}^{\prime}\right) \sigma_{2}^{\prime}\left(a-A^{\prime}\right)}-\right. \\
& D_{6}=\left[\frac{\left(1-B^{\prime}\right) d_{1}\left(1-A^{\prime}\right)}{\left(\sigma_{2}^{\prime}-\sigma_{1}^{\prime}\right) \sigma_{1}^{\prime 2}}\left[\begin{array}{l}
\sum_{k=1}^{\infty} \bar{f}_{U_{1}}^{\prime}\left(\sigma_{1}^{\prime}\right) \prod_{j=2}^{k} \bar{f}_{U_{j}}\left(\frac{\sigma_{1}^{\prime}}{a^{j-1}}\right)+\bar{f}_{U_{2}}\left(\frac{\sigma_{1}^{\prime}}{a}\right)\left(\frac{1}{a}\right) \prod_{j=1, j \neq 2}^{k} \bar{f}_{U_{j}}\left(\frac{\sigma_{1}^{\prime}}{a^{j-1}}\right)+. . \\
++\bar{f}_{U_{k}}^{\prime}\left(\frac{\sigma_{1}^{\prime}}{a^{k-1}}\right)\left(\frac{1}{a^{k-1}}\right) \prod_{j=1}^{k-1} \bar{f}_{U_{j}}\left(\frac{\sigma_{1}^{\prime}}{a^{j-1}}\right)
\end{array}\right] A^{k-1}\right. \\
& +\frac{\left(1-B^{\prime}\right) d_{1}\left(1-A^{\prime}\right)}{\left(\sigma_{2}^{\prime}-\sigma_{1}^{\prime}\right) \sigma_{2}^{\prime 2}}\left[\begin{array}{l}
\sum_{k=1}^{\infty} \bar{f}_{U_{1}^{\prime}}^{\prime}\left(\sigma_{2}^{\prime}\right) \prod_{j=2}^{k} \bar{f}_{U_{j}}\left(\frac{\sigma_{2}^{\prime}}{a^{j-1}}\right)+\bar{f}_{U^{2}}\left(\frac{\sigma_{2}^{\prime}}{a}\right)\left(\frac{1}{a}\right) \prod_{j=1, j \neq 2}^{k} \bar{f}_{U_{j}}\left(\frac{\sigma_{2}^{\prime}}{a^{j-1}}\right)+. . \\
++\bar{f}_{U_{k}} \cdot\left(\frac{\sigma_{2}^{\prime}}{a^{k-1}}\right)\left(\frac{1}{a^{k-1}}\right) \prod_{j=1}^{k-1} \bar{f}_{U_{j}}\left(\frac{\sigma_{2}^{\prime}}{a^{j-1}}\right)
\end{array}\right] A^{k-1} \\
& \bar{f}^{\prime}(0)=-\left(\frac{P_{1}}{\lambda_{1}}+\frac{\left(1-P_{1}\right)}{\lambda_{2}}\right) ; \bar{f}_{U_{i}}{ }^{\prime}\left(\frac{\sigma_{j}}{a^{i-1}}\right)=-\left(\frac{P_{1} \lambda_{1}}{\left(\lambda_{1}+\left(\frac{\sigma_{j}}{a^{i-1}}\right)\right)^{2}}+\frac{\left(1-P_{1}\right) \lambda_{2}}{\left(\lambda_{2}+\left(\frac{\sigma_{j}}{a^{i-1}}\right)\right)^{2}}\right), \mathrm{j}=1,2 ; \mathrm{i}=1,2, \ldots \mathrm{k} ;
\end{aligned}
$$$$
\bar{f}_{U_{i}}^{\prime}\left(\frac{\sigma^{\prime} j}{a^{i-1}}\right)=-\left(\frac{P_{1} \lambda_{1}}{\left(\lambda_{1}+\left(\frac{\sigma^{\prime} j}{a^{i-1}}\right)\right)^{2}}+\frac{\left(1-P_{1}\right) \lambda_{2}}{\left(\lambda_{2}+\left(\frac{\sigma^{\prime} j}{a^{i-1}}\right)\right)^{2}}\right), \mathrm{j}=1,2 ; \mathrm{i}=1,2, \ldots \mathrm{k}
$$$$
\bar{f}_{U_{i}}\left(\frac{\sigma_{j}}{a^{i-1}}\right)=\left(\frac{P_{1} \lambda_{1}}{\left(\lambda_{1}+\left(\frac{\sigma_{j}}{a^{i-1}}\right)\right)}+\frac{\left(1-P_{1}\right) \lambda_{2}}{\left(\lambda_{2}+\left(\frac{\sigma_{j}}{a^{i-1}}\right)\right)}\right), \mathrm{j}=1,2 ; \mathrm{i}=1,2, \ldots \mathrm{k}
$$$$
\bar{f}_{U_{i}}\left(\frac{\sigma^{\prime} j}{a^{i-1}}\right)=\left(\frac{P_{1} \lambda_{1}}{\left(\lambda_{1}+\left(\frac{\sigma_{j}^{\prime} j}{a^{i-1}}\right)\right)}+\frac{\left(1-P_{1}\right) \lambda_{2}}{\left(\lambda_{2}+\left(\frac{\sigma^{\prime} j}{a^{i-1}}\right)\right)}\right), \mathrm{j}=1,2 ; \mathrm{i}=1,2, \ldots \mathrm{k}
$$

Now, differentiating twice with respect to $\mathrm{s}$, the Laplace transform of the density function of time to recruitment at $\mathrm{s}=0$, second moment of $\mathrm{W}$ is determined.

$\mathrm{E}\left(\mathrm{W}^{2}\right)=\frac{\theta_{B}}{\theta_{B}-\theta_{E}} D_{5}{ }^{2}-\frac{\theta_{E}}{\theta_{B}-\theta_{E}} D_{6}{ }^{2}$

where 


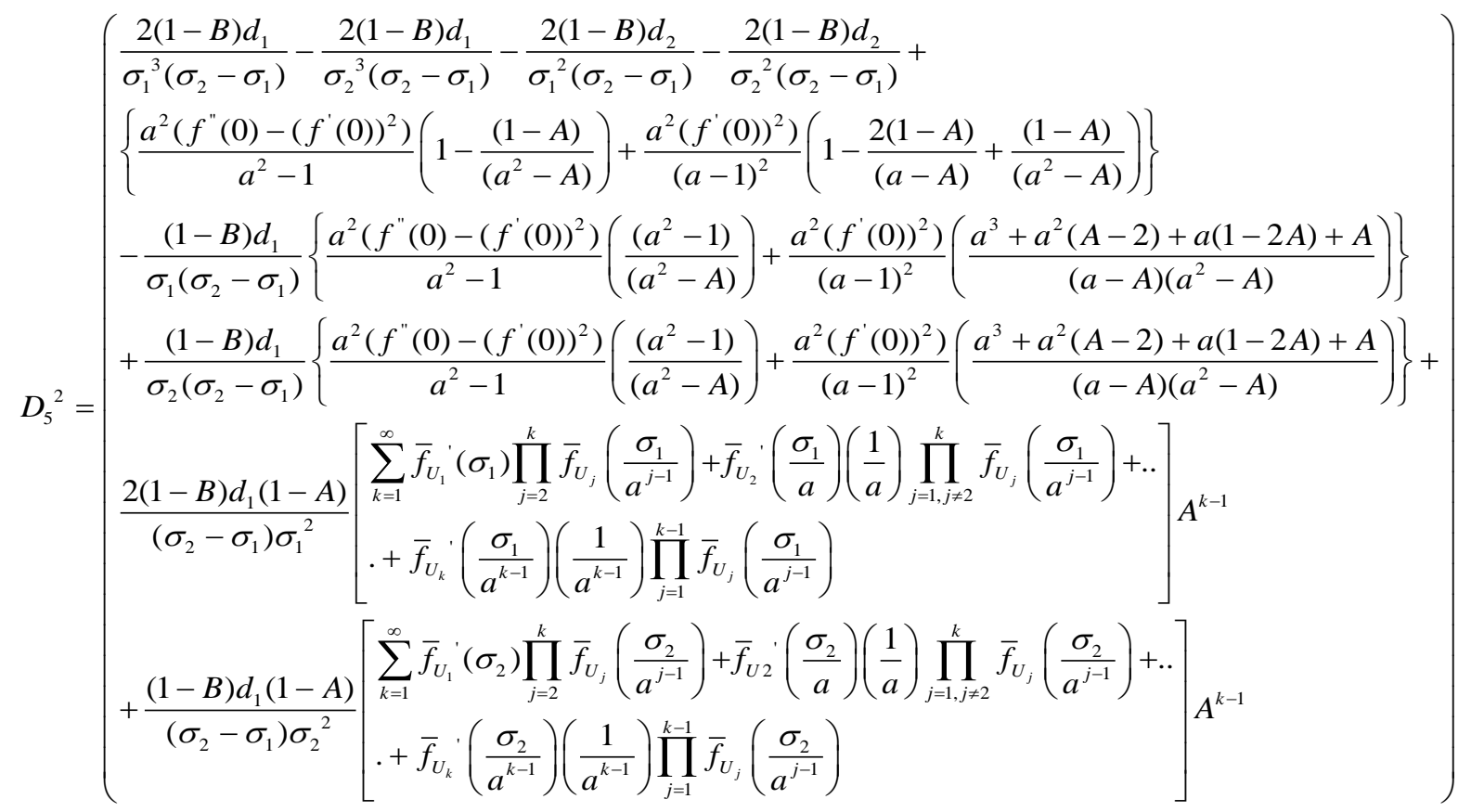

and

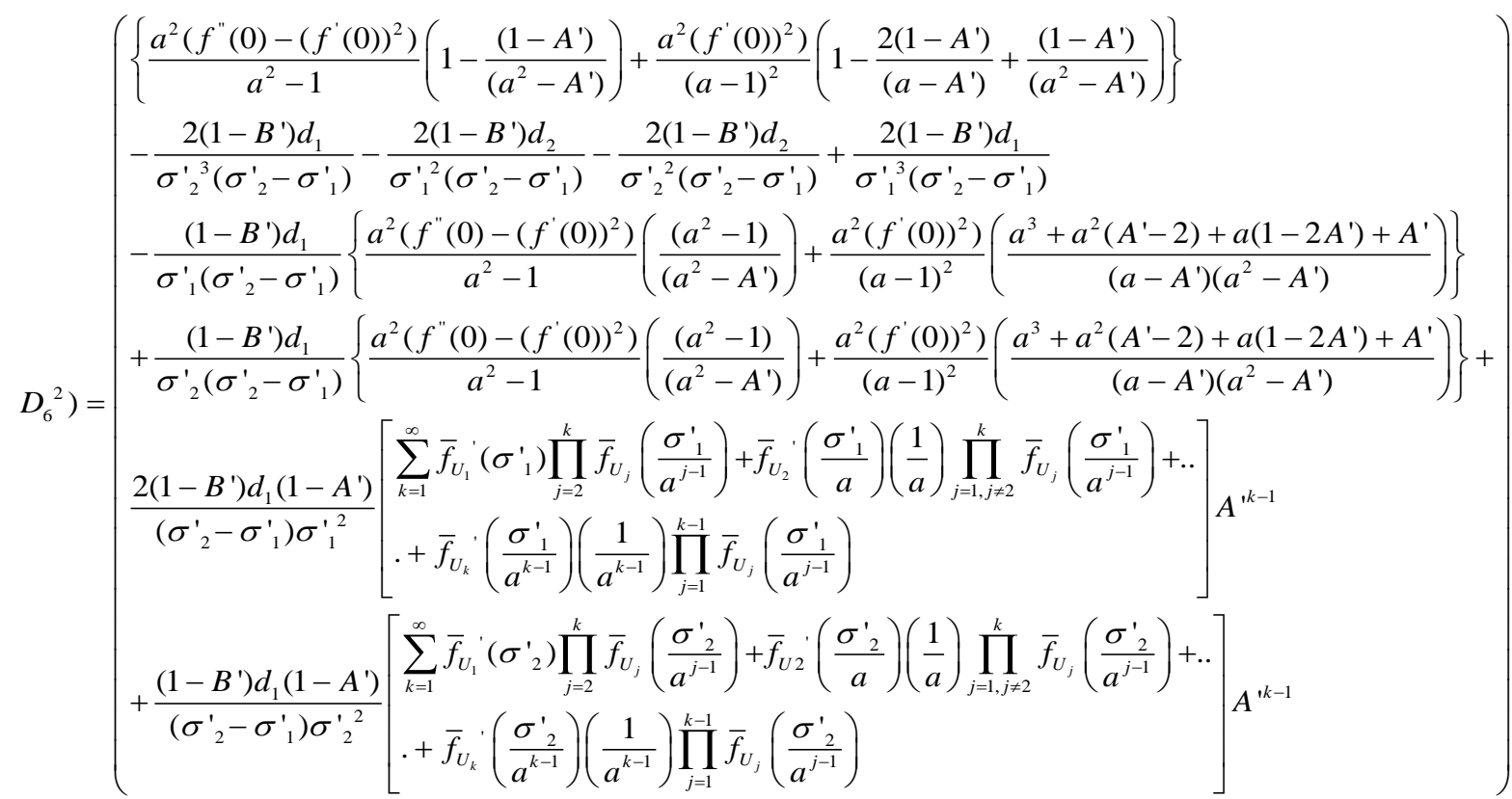

Using equations (6) and (7), the variance of time to recruitment for Case-III is determined.

The results for the notations used in all the three cases are given below:

$$
\begin{array}{ll}
\mathrm{A}=\bar{f}_{X}\left(\theta_{E}\right)=\frac{P_{2} \alpha_{1}}{\alpha_{1}+\theta_{E}}+\frac{\left(1-P_{2}\right) \alpha_{2}}{\alpha_{2}+\theta_{E}} ; & A^{\prime}=\bar{f}_{X}\left(\theta_{B}\right)=\frac{P_{2} \alpha_{1}}{\alpha_{1}+\theta_{B}}+\frac{\left(1-P_{2}\right) \alpha_{2}}{\alpha_{2}+\theta_{B}} ; \\
\mathrm{B}=\bar{g}_{Y}\left(\theta_{E}\right)=\frac{\gamma}{\gamma+\theta_{E}} ; & B^{\prime}=\bar{g}_{Y}\left(\theta_{E}\right)=\frac{\gamma}{\gamma+\theta_{B}} ; \\
\mathrm{b}_{1}=\mathrm{P}_{1} \lambda_{1} \lambda_{2}+\left(1-\mathrm{P}_{1}\right) \lambda_{1} \lambda_{2} ; & \mathrm{d}_{1}=\mathrm{P}_{3} \beta_{1} \beta_{2}+\left(1-\mathrm{P}_{3}\right) \beta_{1} \beta_{2} ; \\
\mathrm{b}_{2}=\mathrm{P}_{1} \lambda_{1}+\left(1-\mathrm{P}_{1}\right) \lambda_{2} ; & \mathrm{d}_{2}=\mathrm{P}_{3} \beta_{1}+\left(1-\mathrm{P}_{3}\right) \beta_{2} ; \\
\mathrm{b}_{3}=\lambda_{1}+\lambda_{2}-A \mathrm{P}_{1} \lambda_{1}-A\left(1-\mathrm{P}_{1}\right) \lambda_{2} ; & \mathrm{d}_{3}=\beta_{1}+\beta_{2}-A \mathrm{P}_{3} \beta_{1}-A\left(1-\mathrm{P}_{3}\right) \beta_{2} \\
b_{3}^{\prime}=\lambda_{1}+\lambda_{2}-A^{\prime} \mathrm{P}_{1} \lambda_{1}-A^{\prime}\left(1-\mathrm{P}_{1}\right) \lambda_{2} ; & d^{\prime}{ }_{3}=\beta_{1}+\beta_{2}-A^{\prime} \mathrm{P}_{3} \beta_{1}-A^{\prime}\left(1-\mathrm{P}_{3}\right) \beta_{2} \\
\mathrm{~b}_{4}=\lambda_{1} \lambda_{2}-A \mathrm{P}_{1} \lambda_{1} \lambda_{2}-A\left(1-\mathrm{P}_{1}\right) \lambda_{1} \lambda_{2} ; & \mathrm{d}_{4}=\beta_{1} \beta_{2}-A \mathrm{P}_{3} \beta_{1} \beta_{2}-A\left(1-\mathrm{P}_{3}\right) \beta_{1} \beta_{2}
\end{array}
$$




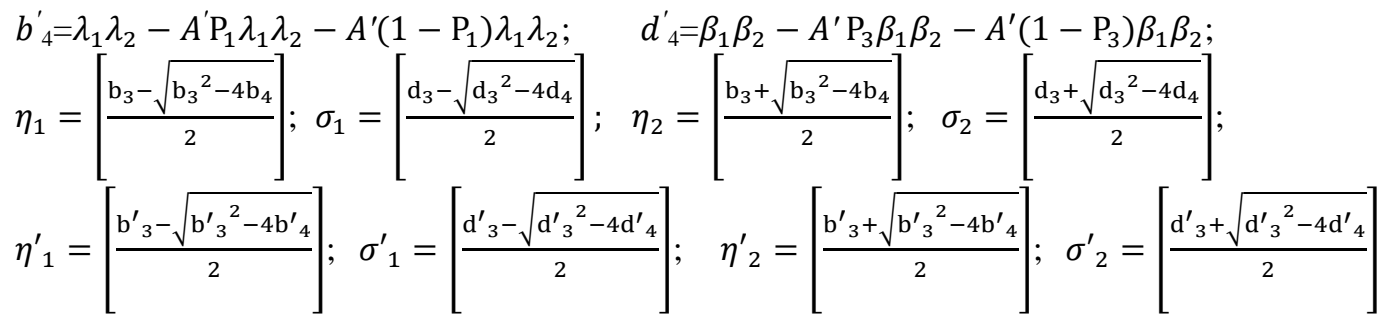

Numerical Illustration:-

The influence of nodal parameters on the performance measures namely mean and variance of the time to recruitment is studied numerically using MATLAB. The performance measures are calculated for all the three cases.

\section{Case-I:}

Effect of $\alpha_{1}$ on the performance measures by fixing the value of the parameters $\mathrm{P}_{1}=0.7 ; \lambda_{2}=0.07 ; \lambda_{1}=0.06 ; \alpha_{2}=0.3$; $\mathrm{P}_{2}=0.6 ; \beta_{1}=0.7 ; \beta_{2}=0.6 ; \mathrm{P}_{3}=0.6 ; \theta_{B}=0.008 ; \theta_{E}=0.04 ; \gamma=0.4$ is given below.

Table-(i)

\begin{tabular}{|c|c|c|}
\hline$\alpha_{1}$ & $\mathrm{E}(\mathrm{W})$ & $\mathrm{V}(\mathrm{W})$ \\
\hline 0.02 & 90.3321 & $1.1605 \times 10^{4}$ \\
\hline 0.023 & 74.3498 & $1.1205 \times 10^{4}$ \\
\hline 0.026 & 61.4058 & $1.0884 \times 10^{4}$ \\
\hline 0.029 & 50.6964 & $1.0623 \times 10^{4}$ \\
\hline 0.032 & 41.6812 & $1.0408 \times 10^{4}$ \\
\hline 0.035 & 33.9826 & $1.0229 \times 10^{4}$ \\
\hline
\end{tabular}

\section{Case-II:}

Effect of $\rho$ on the performance measures by fixing the value of the parameters $\alpha_{1}=0.9 ; \alpha_{2}=0.1 ; \mathrm{P}_{2}=0.06 ; \beta_{1}=0.6$; $\beta_{2}=0.7 ; \mathrm{P}_{3}=0.06 ; \theta_{B}=0.009 ; \theta_{E}=0.09 ; \lambda=18 ; \gamma=0.7 ; \mathrm{k}=5$ is given below.

Table-(ii)

\begin{tabular}{|l|l|l|}
\hline \multicolumn{1}{|c|}{$\rho$} & $\mathrm{E}(\mathrm{W})$ & $\mathrm{V}(\mathrm{W})$ \\
\hline 0.92 & 1.3479 & $7.9179 \times 10^{3}$ \\
\hline 0.921 & 4.6516 & $7.1472 \times 10^{3}$ \\
\hline 0.922 & 8.0855 & $6.3230 \times 10^{3}$ \\
\hline-0.348 & 31.0744 & $1.6249 \times 10^{3}$ \\
\hline-0.349 & 31.3255 & $1.5897 \times 10^{3}$ \\
\hline-0.35 & 31.5767 & $1.5523 \times 10^{3}$ \\
\hline
\end{tabular}

\section{Case-III:}

Effect of ' $a$ ' on the performance measures by fixing the value of the parameters $\mathrm{P}_{1}=0.006 ; \lambda_{2}=0.05 ; \lambda_{1}=0.03$; $\alpha_{2}=0.07 ; \mathrm{P}_{2}=0.08 ; \beta_{1}=0.5 ; \beta_{2}=0.7 ; \mathrm{P}_{3}=0.47 ; \theta_{B}=0.008 ; \theta_{E}=0.07 ; \gamma=0.005 ; \mathrm{k}=3 ; \alpha_{1}=0.04$ is given below.

Table-(iii)

\begin{tabular}{|l|l|l|}
\hline \multicolumn{1}{|c|}{$a$} & $\mathrm{E}(\mathrm{W})$ & $\mathrm{V}(\mathrm{W})$ \\
\hline 0.007 & 126.1315 & 86.1716 \\
\hline 0.0072 & 126.5648 & 86.2258 \\
\hline 0.0074 & 127.0011 & 86.2766 \\
\hline 0.0076 & 127.4404 & 86.3242 \\
\hline 0.0078 & 127.8829 & 86.3689 \\
\hline 0.008 & 128.3285 & 86.4109 \\
\hline
\end{tabular}




\section{Conclusion:-}

i) For the Case-(i), if the value of $\alpha_{1}$ increases then the mean time to recruitment and the variance of time to recruitment decreases.

ii) For the Case-(ii), if the positive value of $\rho$ increases then the mean time to recruitment increases and the variance of time to recruitment decreases, if the negative value of $\rho$ decreases then the mean time to recruitment increases and the variance of time to recruitment decreases.

iii) For the Case-(iii), if the value of the parameter $a$ increases then the mean time to recruitment and the variance of time to recruitment increases.

iv) Since the time to recruitment is more elongated in Case-III than the first two cases, Case-III ispreferable from the organization point of view.

\section{References:-}

1. Bartholomew, D.J. (1969). Renewal theory models for manpower Systems in N. A. B. Wilson, 120-128.

2. Bartholomew, D.J. (1971). The statistical approach to manpower planning. Statistician, No: 20, 3-26.

3. Bartholomew, D.J. and Forbes, A. F. (1979). Statistical techniques for manpower planning. John Wiley, Chichester.

4. Clough, D.J., Lewis, C. G. and Oliver, A.L. (1974). Manpower planning models. English University Press, London.

5. Devi, A. and Srinivasan, A. (2014). Probabilistic analysis on time to recruitment for a single grade manpower system with different epochs for decisions and exits. International Journal of Revolution in Science and Humanity, 4(2), 59-64.

6. Esary, J. D., Marshall, A. D. and Proschan. (1973). Shock models and wear processes. The Annuals of Probability, Vol. No:4, 627-649.

7. Grinold, R. C. and Marshall, K. T. (1977). Manpower planning models. North-Holland, New York.

8. Kasturri, K. (2007).Mean time for recruitment and cost analysis on some univariate policies of recruitment in manpower models. Ph. D., Thesis, Bharathidasan University.

9. Mariappan, P. (2002). Study of some manpower models and their analysis. Ph.D., Thesis, Bharathidasan University.

10. Saavithri, V. (2003). Cost analysis on some policies of recruitment in manpower models. Ph.D., Thesis, Bharathidasan University.

11. Sathiyamoorthi, R. and Elangovan, R. (1998). Optimal time interval between recruitment programmes. International Journal of Management and Systems, 15(2), 117-128.

12. Sathyamoorthi, R. and Parthasarathy. S. (2003). On some stochastic models for manpower planning using SCBZ property. Ph. D., Thesis at Department of Statistics, Annamalai University.

13. Uma, K. P. (2010). A study on manpower models with univariate and bivariate policies of recruitment. Ph. D., Thesis, Avinashilingam University for Women. 